Scientific Paper

\title{
Secured healthcare monitoring system in wireless body area network using polynomial based technique
}

\author{
Shanthapriya $\mathrm{R}^{1, \mathrm{a}}$, Vaithianathan $\mathrm{V}^{1}$ \\ ${ }^{I}$ Department of ECE, SSN College of Engineering, Kalavakkam-603110, Chennai, Tamilnadu, India \\ ${ }^{a}$ E-mail address: shanthaarona@gmail.com
}

(received 19 February 2019; accepted 27 June 2019)

\begin{abstract}
Nowadays healthcare is a standout amongst the most rapidly developing application zone in body area networks (BANs). BANs are anticipated to play a significant role in the stream of patient-health monitoring. To provide, secure BAN access to the entire system without disturbing the confidentiality of patients' therapeutic data is a major challenge. Security of the BAN can be improved by using a polynomial curve-based steganography technique. The performance of the proposed BAN is evaluated by using a Fourier transform and Wavelet transform. The results of the proposed BAN are presented and compared for both the transforms.
\end{abstract}

Key words: healthcare; body area networks; confidentiality; security; steganography; polynomial curve; transforms.

\section{Introduction}

The last couple of decades have seen a relentless increment in various parts of the world prompting a sharp ascent in the number of elderly individuals. A current report from the United Nations [1] anticipated that there will be 2 billion (22\% of the total populace) more seasoned individuals by 2050 . Likewise, research shows that around $89 \%$ of aged individuals are probably going to live autonomously. However, medicinal research found that around $80 \%$ of the aged individuals older than 65 experiences at least one endless malady [2-5]. Accordingly, giving a nice personal satisfaction for aged individuals has turned into a genuine social challenge. The rapid multiplication of data and communication technologies is empowering creative healthcare arrangement and devices that show guarantee in addressing the aforementioned challenges.

Body Area Network (BAN) permits the integration of astute, small-sized low-power sensor hubs in, on or nearby human body to screen body capacities and the encompassing condition. It can possibly reform the eventual fate of human services and accomplish various analysts both from the scholarly world and industry in a previous couple of years. For the most part, BAN comprises in-body and on-body sensor systems. In-body sensor permits the correspondence between intrusive/embedded gadgets and base station. However, the onbody sensor permits the correspondence between nonobtrusive/wearable gadgets and a facilitator [6-11]. Each sensor in the BANs is integrated with biosensors to collect vital signs like Electrocardiogram (ECG), Blood Pressure (BP), Electroencephalography (EEG), etc. The gathered physiological signs are forwarded to a facilitator called controller which can be a compact gadget, such as smartphone, PDA, etc. In addition, when the controller distinguishes any variations from the norm then it gives the prompt alarm to the individual wearing the bio-sensors. For instance, a normal level of BP in an individual should not be less than or equivalent to 120. If the BP of an individual goes beyond 126 , the controller will give a delicate caution to the individual (e.g. beep tone) is shown in Figure 1.

Security plays the most important role in any system. Individuals have a different point of view with respect to security and consequently, it is projected in various perspectives. In general, communication in sensor-related network applications (like BAN) in medicinal services are usually wireless in nature. This may result in different security issues to these frameworks. In this part, we portray the key security prerequisites in healthcare system utilizing BAN.

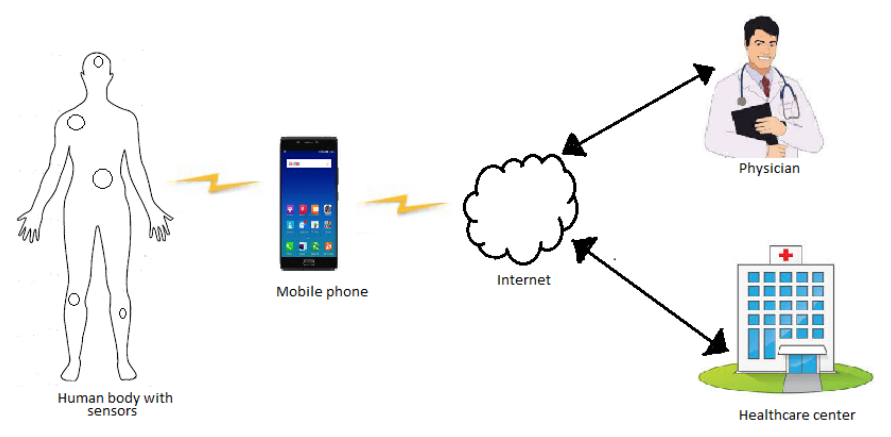

Figure 1.Body Area Network Architecture 


\section{Data privacy}

Like WSNs, the most essential issue in BAN is data privacy. It is vital to shield the information from disclosure. BAN system should not release the patient's imperative data to outside or neighboring systems. In a healthcare application, the sensor hubs gather and forward the sensitive information to a controller. An adversary can listen stealthily on the communication and can catch basic information. This kind of eavesdropping may cause extreme harm to the patient since the foe can utilize the acquired information for many purposes.

\section{Data integrity}

Integrity is an important aspect in WBAN because an adversary can simply modify the information by including a few pieces of false data and forward the altered information to the controller. Absence of integrity is very dangerous during a life-critical situation (when emergency information is changed)

\section{Data Authenticity}

Authenticity is an affirmation of the identities of conveying hubs. Each hub has to realize that the received data originates from a genuine sender. Or else, the receiving hub can be duped into playing out some wrong activities.

\section{Data freshness}

All information collected from the receiver must be valid for a constrained time interval. Accordingly, when a hub gets data, it should be guaranteed that the data is new. Otherwise, the received information is pointless because the conveyed information is invalid.

Patient monitoring becomes more feasible due to the advancement of the BAN in healthcare applications. Recently several projects and wireless healthcare researches have been developed which aims to provide persistent patient monitoring. This section depicts a few popular research works about healthcare system utilizing body area networks.

Krishna K. Venkatasubramanian [12] proposed PSKA (physiological-signal-based key agreement) scheme, a plan for empowering secure inter sensor correspondence inside a BAN in a usable (plug-n-play, straightforward) way. PSKA enables neighboring hubs in BAN to allow the shared symmetric cryptographic key in a validated way, utilizing physiological signs acquired from the subject. No initialization or preorganization was required; simply conveying sensors in a BAN is sufficient to influence them to impart safely.

Wei Wang in [13] proposed the IPI (inter-pulse interval) signal design at transmitter side was considered as a biometric verification key utilizing Gaussian mixture model (GMM). A light-weight signature confirmation scheme was adopted in the receiver side that utilizes IPI signals. High sampling misalignment resistance is the advantage of the proposed authentication scheme. The major commitment of this GMM approach is to apply stochastic pattern acknowledgment to ECG signal which provides security, so it is different from other traditional methodologies.

Mehmet R. Yuce in [14] depicts the complete usage of a WBAN framework to deploy in the therapeutic environment. Issues related to hardware executions, programming, and remote protocol plans are addressed effectively. In addition, current issues and new innovations related to continuous monitoring of the patients are also explained briefly. In this paper, the multi-hopping technique is used in a WBAN framework that can be utilized as a part of restorative conditions for observing the physiological parameters in the wireless medium. The framework is quite different from other existing techniques by considering a scenario that the vital signs are observed from numerous patients at the same time in the real-time application.

In 2006, Chakravorty designed a project called Mobicare [15] specially developed for healthcare monitoring. It provides a wide-area portable patient monitoring framework that encourages nonstop and opportune checking of the patient's physiological status. Despite, the author recognized the security issues in MobiCare, but it was not sufficient for continuous healthcare applications. In this manner, security and protection are still not executed in MobiCare or may have been overlooked for future work. However, there are numerous security issues such as secure restriction, obscurity, and so on, are not included in MobiCare system.

\section{Methods}

The improvement in wireless BAN plays an important role in healthcare applications. The sensors used in healthcare are equipped for gathering vital signs from various parts of the body. The gathered signals are processed and forwarded to the controller. A WBAN is an arrangement of biosensors planned to convey the information to remote clients. Advances in new developments and communication have changed the remedial system from paper content to electronic information.

The mobile healthcare system gives amazing flexibility to patients and social insurance providers. The outline of WBAN is exceptionally complex because the vital medical information is traded to remote clients through the internet. In this section, the work is initiated towards patient monitoring. The association of sensor nodes should satisfy all protection prerequisites without eliminating the security and privacy measures, or else it causes major trouble to patients. The recent advances in wireless communication permit the sensor (wearable or implantable) to trade information in wireless medium to the base station without affecting the everyday activity of a person. The following Figure 2 shows the schematic outline for secure health monitoring in WBAN. 


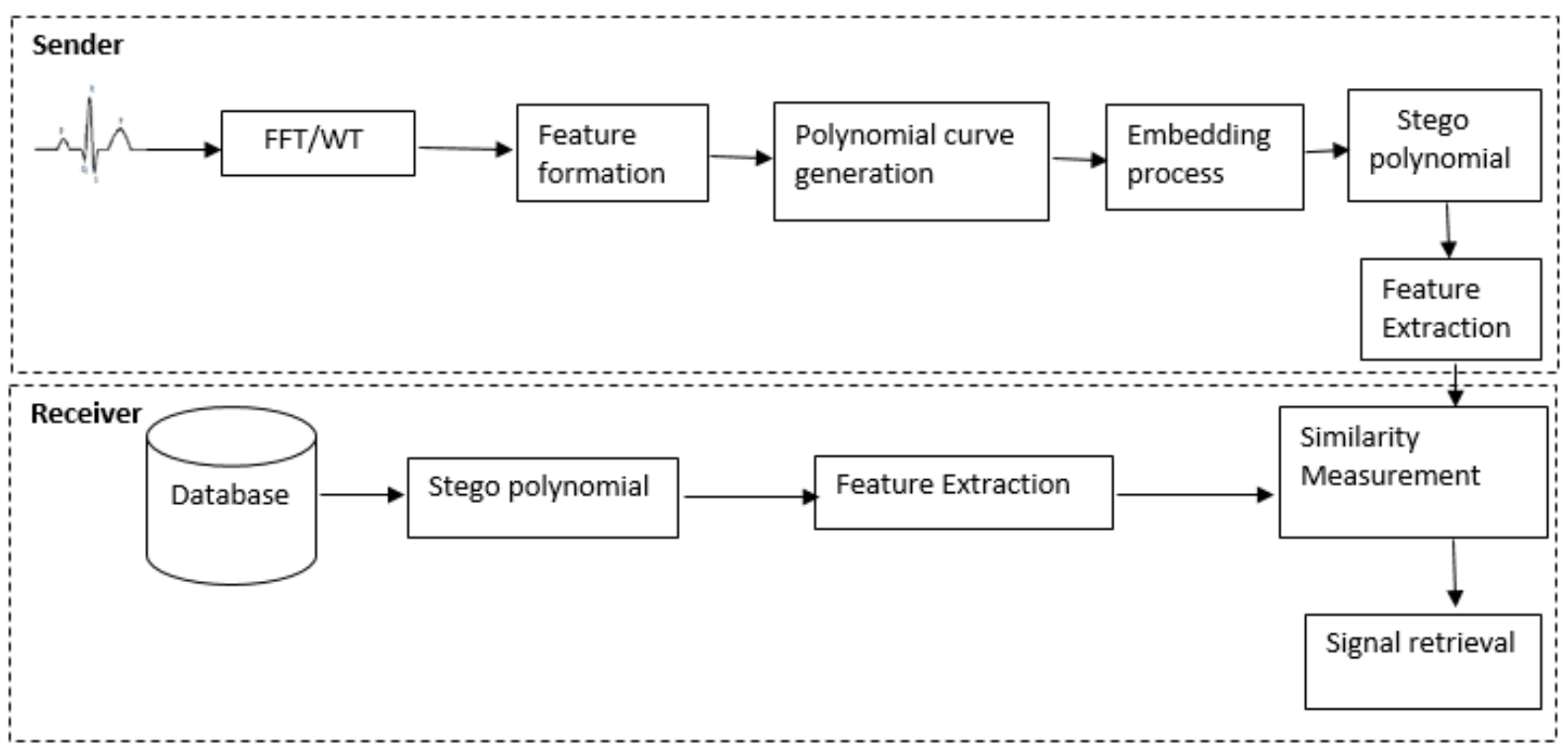

Figure 2. Schematic outline for the secure healthcare system

\section{Polynomial Based curve generation}

The goal is to develop a secure health-monitoring framework in WBAN that brings confidentiality and authenticity without disturbing the privacy of the patient. To attain security, a higher-order polynomial is utilized to create a curve. Polynomial based curve is used because there is no unequivocal formula when the order of the polynomial is higher.

For this reason, the concept of the polynomial based curve is used which installs secret information into the curve and makes the data secure so that the hacker cannot access the data or manipulate it and hence the patient's secret information is protected. In general, the nth order of the polynomial is in the form of $\mathrm{P}(\mathrm{x})=\mathrm{g}^{\mathrm{n}} \mathrm{x}^{\mathrm{n}}+\mathrm{g}^{\mathrm{n}-1} \mathrm{x}^{\mathrm{n}-1}+\ldots+\mathrm{g}^{0}$ where $\mathrm{n}$ represents the polynomial order. To generate the polynomial curve, the accompanying steps must proceed:

1. considering $x$ is a linearly spaced vector $[a, b]$ in an interval and $y$ can be a function (tan, cos, or sin) or values.

2. the coefficients of the polynomial is generated by using polyfit $(\mathrm{x}, \mathrm{y}, \mathrm{n})$ function where $\mathrm{x}$ and $\mathrm{y}$ represent the length of the interval and function respectively, where $\mathrm{n}$ denotes the polynomial order.

3. the obtained coefficients are integrated to form an equation. Using polyval () function it can be evaluated to generate a polynomial curve.

By following the above steps, the equation formed for generating a polynomial curve is given by

$P(x)=0.6981 x^{5}+1.3963 x^{4}+2.5094 x^{3}+4.3795 x^{2}-$

$1.3886 x+0.9581$

Eq. 1

The equation can be evaluated and plotted as a polynomial curve using polyval() function.

\section{Steganography}

Steganography is the method of covered communication and an effort to hide the presence of the embedded data. The unique message is being covered up inside a carrier signal with the end goal that the progressions so occurred in the carrier signal are not perceptible. It is one of the best methods to provide security and protection to the secret data without revealing it to the neighboring hubs or intruders. Steganography algorithm is used as a hiding technique to embed the confidential information into the created polynomial curve. Hidden polynomial curve (Stego polynomial) looks slightly similar to the generated polynomial curve to protect the secure data from hackers.

\section{Sender}

The input signal (ECG signal) is taken from the MIT-BIH database. The signal is converted from the time domain to frequency domain by applying FFT. The signal is quantized to detect peak points. The obtained peak points are transformed into binary form thereby forming a feature vector. The higherorder polynomial curve is generated after the features are formed. Using steganography algorithm, the polynomial based curve embeds the feature vector into it forming stego Polynomial.

\section{Receiver}

All information about the patient and their vital signs are stored in the dataset. Similarity measurement is done as a part of the checking process. The features from the sender and receiver are extracted for similarity checking. If a match arises between them, then the information will be intimated to the physician along with the patient's medical data for further analysis. 


\section{Results and Discussion}

\section{ECG Signal}

Electrocardiogram (ECG) signal has been broadly utilized for the determination of numerous heart ailments, the main source of sudden death universally. ECG signal helps to detect abnormalities in the human body easily.

\section{FFT}

Fast Fourier transform is used to convert a signal from the time domain to frequency domain because frequency component of ECG signal, have comparable values at any given time, regardless of where they are estimated on the body. The other advantages of using FFT are:

1. they are stable and easy to detect peak points;

2. they describe a subject's physiological signal extremely well.

After performing FFT, the signal is quantized to convert an expansive arrangement of a large set of values into a small set as shown in Figure 3. The input ECG signal is sampled and rounded during the quantization process. The variation between the quantized signal and the input signal helps in distinguishing the peak points. The acquired signals are changed to binary string and connected together with peak values thereby forming features. The gathered features are assembled to frame feature vector.

\section{WT}

In Wavelet transform, a signal is decomposed into an arrangement of premise capacities called wavelets. These are acquired from a solitary model wavelet, called mother wavelet, by dilatations and withdrawals, and in addition by shift [16]. ECG signal is taken as input signal which is decomposed with symlet (sym) wavelet at depth 3. First, the input ECG signal is loaded into the wavelet program for the decomposition process. The decomposed signal is shown in Figure 4. The loaded signal is examined as matrix form and the signal is decomposed to n-level that deliver $2^{\mathrm{n}}$ distinct arrangements of coefficients. For this work, the estimation of $\mathrm{n}$ is chosen as 5 . The coefficients are linked together to form a feature. Collected features are grouped together to form a feature vector. A polynomial curve is generated to embed the feature values into it and forms stego polynomial.

$a 5=d 5+d 4+d 3+d 2+d 1$

Eq. 2

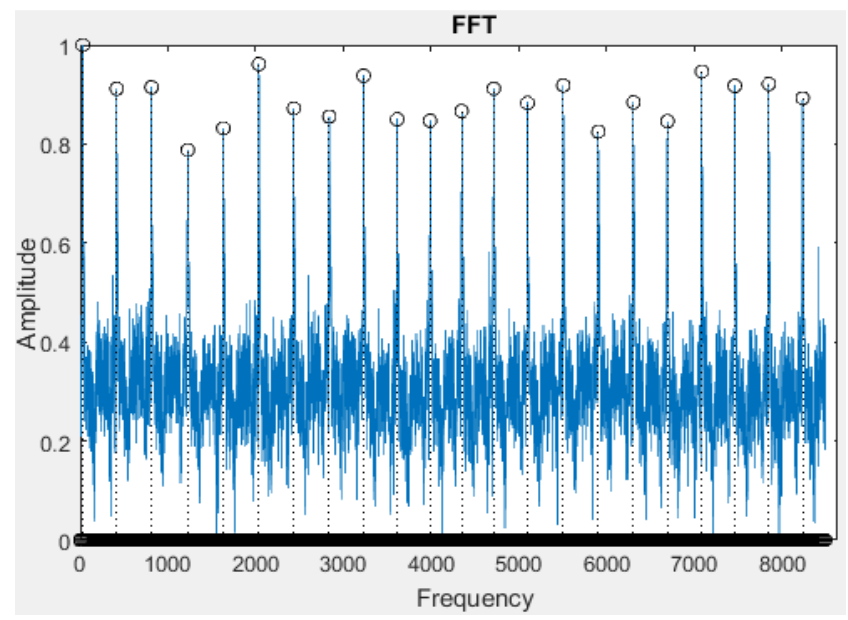

Figure 3. FFT signal

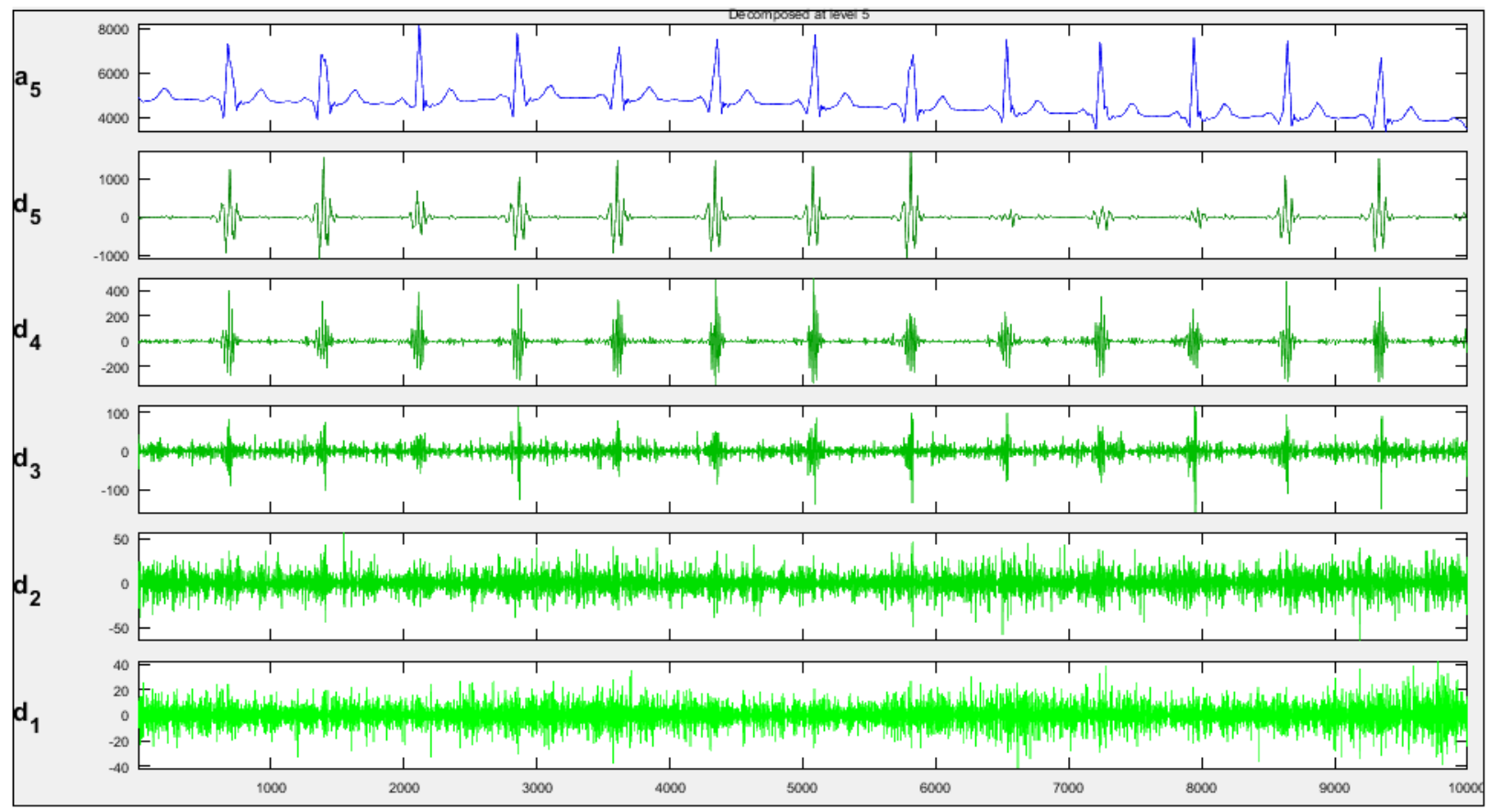

Figure 4. Decomposed signal 


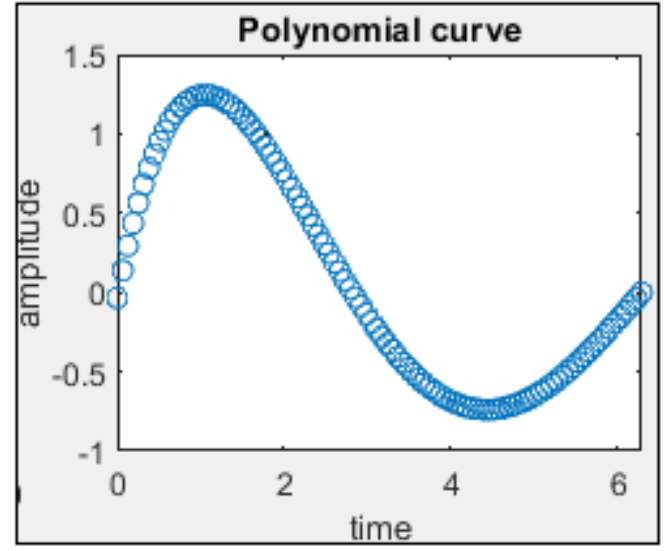

Figure 5a. Generated polynomial curve for FFT

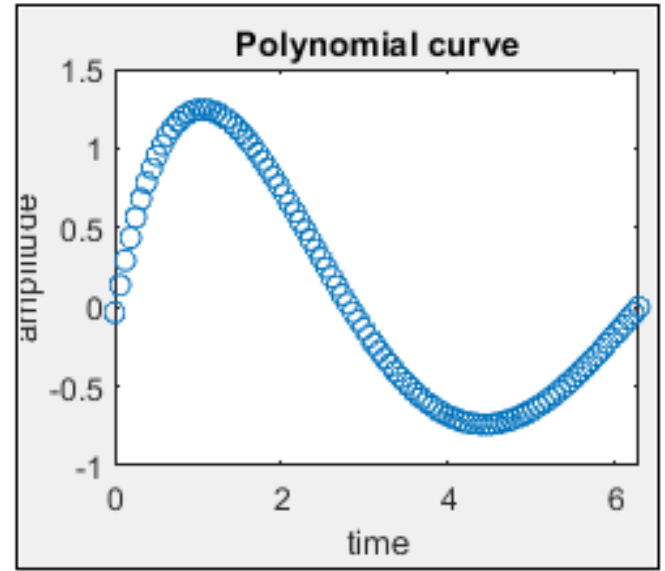

Figure 6a. The generated polynomial curve for WT

\section{Polynomial curve generation and hiding}

After the feature formation process, a polynomial curve is created to shroud the features into it thereby the data inside the curve is sheltered and gives secure transmission as shown in Figure 5a and $\mathbf{5 b}$. Using steganography algorithm, the features are hidden in the generated polynomial to give security to the data, as shown in Figure $\mathbf{6 a}$ and $\mathbf{6 b}$.

\section{Database and checking process}

For this work, ECG signals, taken from the MIT-BIH Arrhythmia databases [17] were utilized. This database, giving an online signal by Physio Net, is an aftereffect of the cooperation between BIMC Centre and MIT, and it is standouts amongst the most used databases for exploring purposes. The input feature values and the loaded data are checked in order to find the similarities. If any match arises a notification regarding "Authenticate" is displayed along with their current ECG signal. If mismatch arises the ECG signal cannot be retrieved and the process stopped immediately with a notification "unauthenticated data" is shown in Figure 7. Retrieved ECG signal after authentication, is shown in Figure 8.

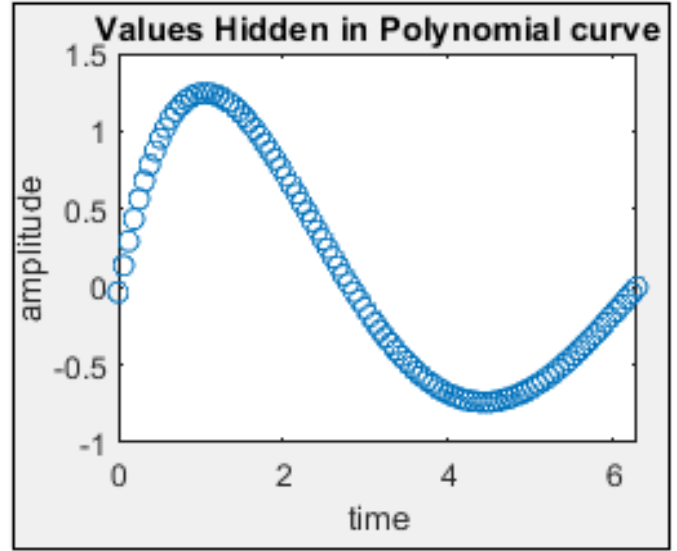

Figure 5b. Values hided into the polynomial curve

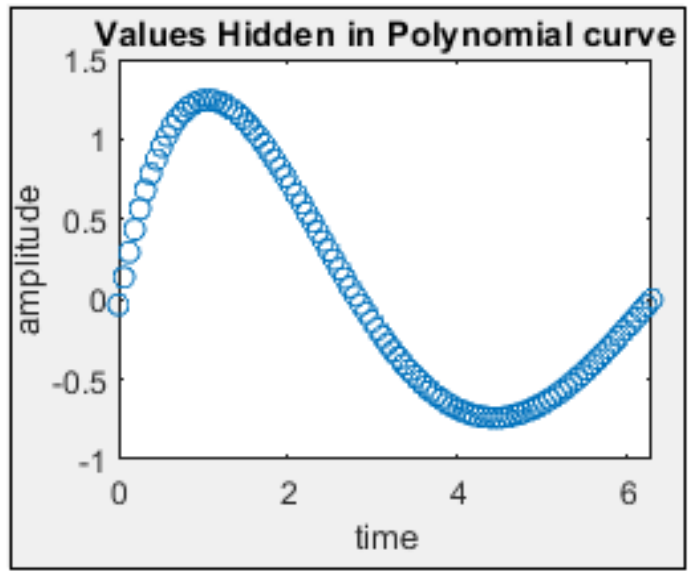

Figure 6b. Values hidden into the polynomial curve
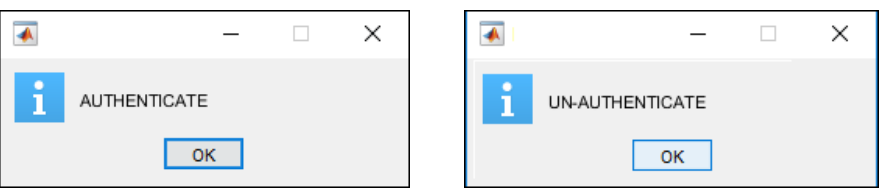

Figure 7. Authenticated and un-authenticated notification

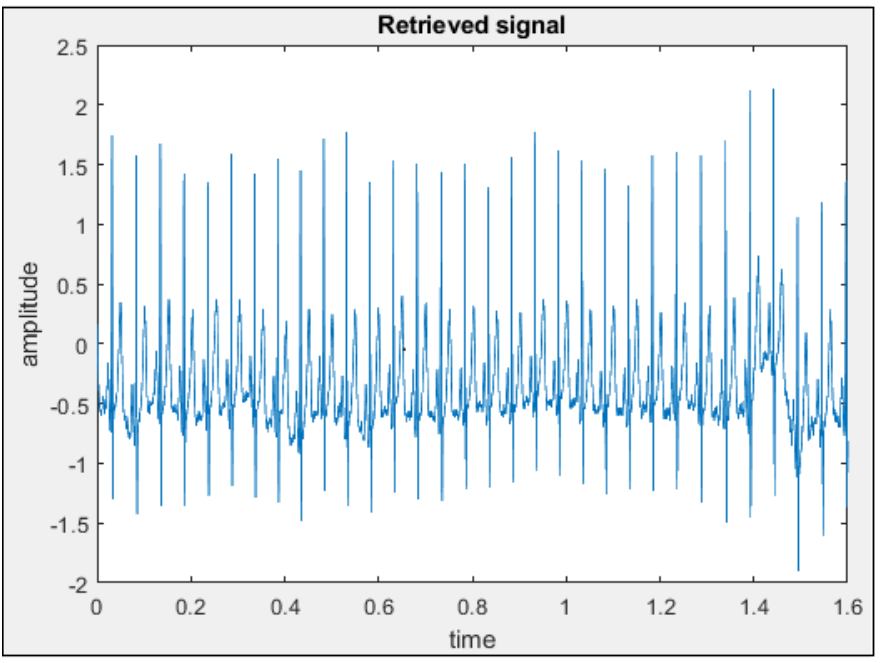

Figure 8. Retrieved signal 
Performance of the system is based on PSNR and MSE value. A system is said to be a better system only if its PSNR value is high and its MSE value is less. PSNR and MSE values can be calculated by

$P N S R=10 * \log _{10}\left(\frac{b^{2}}{M S E}\right)$

Eq. 3

MSE $=\frac{1}{X Y} \sum_{x=1, y=1}^{n}\left[I_{1}(x, y)-I_{2}(x, y)^{2}\right]$

$\mathrm{Eq} \cdot 4$

The PSNR and MSE values of the signal for both FFT and WT transform is shown in Figure 9 and Figure 10. Similarly, for other signals, the PSNR and MSE values are taken and tabulated (see Table 1 and Table 2).

The tabulated values are plotted in the form of a graph, as shown in Figure 11a and Figure 11b. The security of the BAN network is based on the hiding capacity and the order of the

Table 1. PSNR and MSE values for different signals using FFT

\begin{tabular}{ccc}
\hline \hline Signal & PSNR & MSE \\
\hline S1 & 57.1847 & 18.3489 \\
S2 & 42.3493 & 22.4896 \\
S3 & 54.1496 & 16.9103 \\
S4 & 60.9412 & 17.9616 \\
S5 & 74.8163 & 13.0817 \\
S6 & 40.9784 & 19.6502 \\
S7 & 76.8471 & 9.3618 \\
S8 & 45.3891 & 10.7418 \\
S9 & 71.4692 & 12.4136 \\
S10 & 63.5983 & 20.9248 \\
S11 & 41.8175 & 19.1811 \\
S12 & 69.6390 & 22.1512 \\
S13 & 51.1844 & 20.4380 \\
S14 & 62.7150 & 15.1381 \\
S15 & 74.1814 & 18.0143 \\
\hline \hline
\end{tabular}

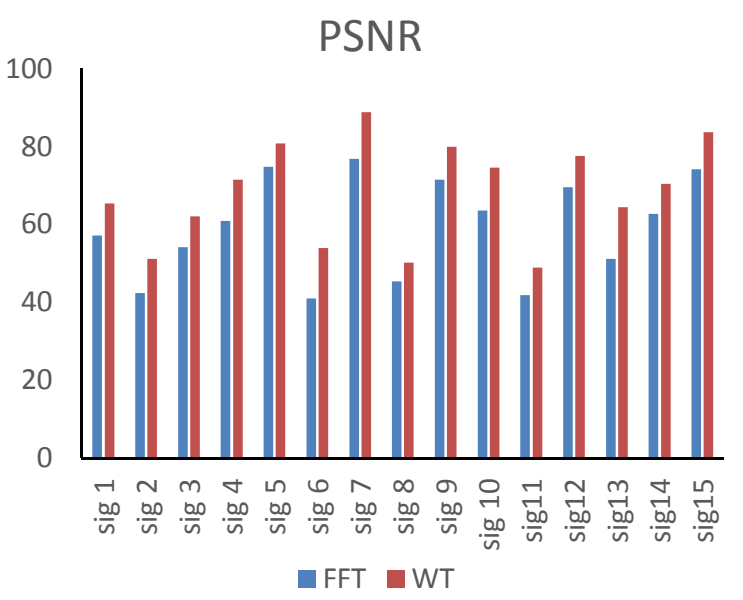

Figure 11a. PSNR Validation graph for FFT and WT polynomial. If the hiding capacity and polynomial order increase the security also increases is shown in Figure 12.

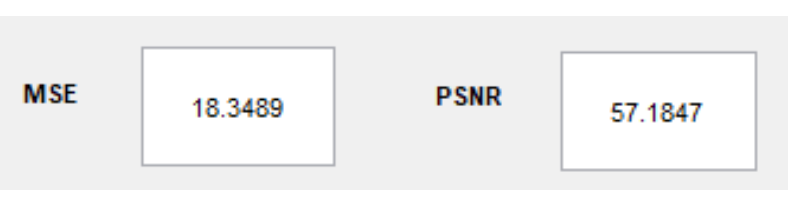

Figure 9. FFT Validation

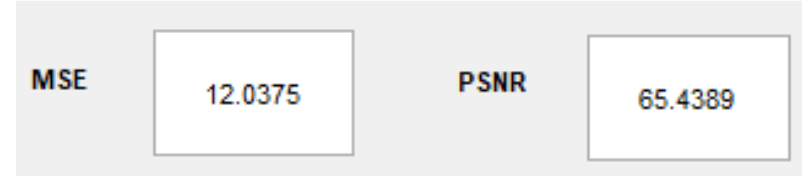

Figure 10. WT validation

Table 2. PSNR and MSE values for different signals using Wavelet transform

\begin{tabular}{ccc}
\hline \hline Signal & PSNR & MSE \\
\hline S1 & 65.4389 & 12.0375 \\
S2 & 51.1907 & 18.4961 \\
S3 & 62.0319 & 10.2936 \\
S4 & 71.5403 & 13.1059 \\
S5 & 80.8372 & 9.0115 \\
S6 & 53.9406 & 15.3166 \\
S7 & 88.8249 & 4.8094 \\
S8 & 50.1653 & 14.9243 \\
S9 & 79.9386 & 9.5314 \\
S10 & 74.6091 & 17.0943 \\
S11 & 48.9633 & 13.0143 \\
S12 & 77.5661 & 16.1248 \\
S13 & 64.4134 & 14.6133 \\
S14 & 70.4417 & 11.1488 \\
S15 & 83.7488 & 10.2723 \\
\hline \hline
\end{tabular}

MSE

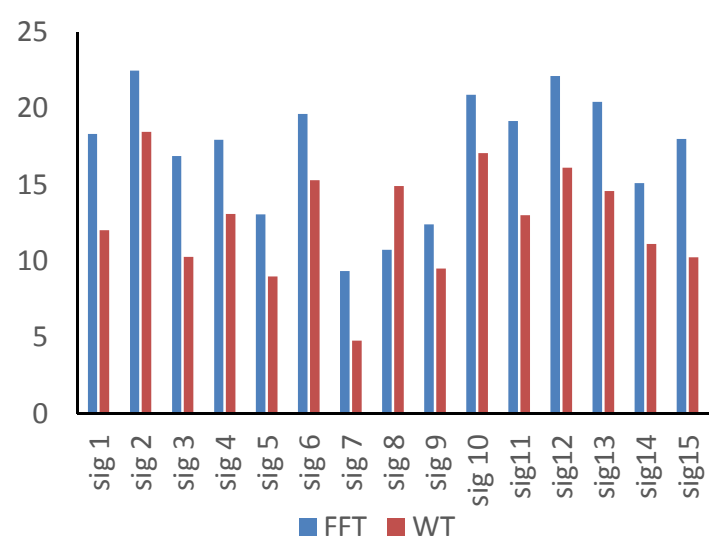

Figure 11b. MSE Validation graph for FFT and WT 


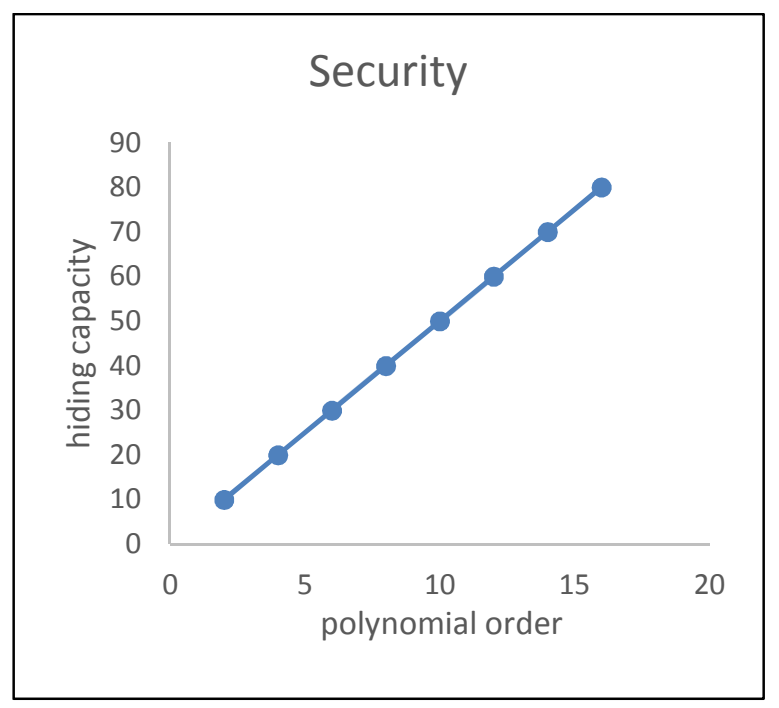

\section{Conclusion}

The secure communication inside the BAN is needed to protect the patient's privacy and security. But providing security in healthcare application without affecting the patient's therapeutic information is still a challenge. In this work, a polynomial curve-based steganography technique has been used for security purpose. Performance metrics like PSNR and MSE are evaluated for FFT and WT. By comparing the results, it is evident that WT gives high PSNR value and less MSE value when compared to FFT.

Figure 12. Security graph

\section{References}

[1] UN (2014), World population ageing 2013, Statistical Papers - United Nations (Ser. A), Population and Vital Statistics Report, UN, New York, https://doi.org/10.18356/30d0966c-en.

[2] Weinstein R. RFID: A technical overview and its application to the enterprise. IT Prof. 2005;7(3)27-33.

[3] Bulling A, Blanke U, Schiele B. A tutorial on human activity recognition using body-worn inertial sensors. ACM Comput Surveys (CSUR). 2014;46(3):33.

[4] Galzarano S, Giannantonio R, Liotta A, Fortino G. A task-oriented framework for networked wearable computing. IEEE Trans Autom Sci Eng. 2016;13(2)621-638.

[5] Watanabe H, Kawarasaki M, Sato A, Yoshida K. Wearable ecg monitoring and alerting system associated with smartphone: iHeart. Int J E-Health Med Commun (IJEHMC). 2013;4(4)1-6.

[6] Milenković A, Otto C, Jovanov E. Wireless sensor networks for personal health monitoring: issues and an implementation. Comput Commun. 2006;29(13):2521-2533.

[7] Otto C, Milenković A, Sanders C, Jovanov E. System architecture of a wireless body area sensor network for ubiquitous health monitoring. J Mob Multimedia. 2006;1(4):307-326.

[8] Movassaghi S, Abolhasan M, Lipman J, et al. Wireless body area networks: a survey. IEEE Commun Surv Tutor. 2014;16,(3):16581686

[9] Gope P, Hwang T. BSN-Care: A secure IOT based modern healthcare system using body sensor network. IEEE Sensors J. 2016: 16(5):1368-1376.

[10] Azariadi D, Tsoutsouras V, Xydis S, Dimitrios D. ECG signal analysis and arrhythmia detection on IoT wearable medical devices. 5th International Conference on Modern Circuits and Systems Technologies (MOCAST), 2016.

[11] Venkatasubramanian KK, Banerjee A, Gupta S. Plethysmogram-based Secure Inter-Sensor Communication in Body Area Networks. In: Proceedings - IEEE Military Communications Conference MILCOM. 2008

[12] Venkatasubramanian KK, Banerjee A, Gupta S. PSKA: Usable and Secure Key Agreement Scheme for Body Area Networks. IEEE Trans Information Technology in Biomedicine. 2010;14(1):60-68.

[13] Wang W, Wang H, Peng D, Sharif H. Secure Stochastic ECG Signals Based on Gaussian Mixture Model for e-Healthcare systems. IEEE Systems Journal. 2011;5(4):564-573.

[14] Yuce MR. Implementation of wireless body area networks for healthcare systems. Sensors and Actuators A: Physical. 2010;162(1):116-129.

[15] Chakravorthy R. A Programmable service architecture for mobile medical care. 4th Annual. IEEE Int Conf Pervasive Comput Commun Workshop (PERSOMW). 2006.

[16] Anbarjafari G Demirel H. Image super resolution based on interpolation of wavelet domain high frequency subbands and the spatial domain input image. ETRI J. 2010:32(3):390-394.

[17] Moody GB, Mark RG. The impact of the mit-bih arrhythmia database. IEEE Eng Med Biol Mag. 2001;20(3):45-50. 